\title{
Heat shock proteins HSP70 and MRJ cooperatively regulate cell adhesion and migration through urokinase receptor
}

\author{
Yuli Lin ${ }^{1 \dagger}$, Nana Peng ${ }^{1 \dagger}$, Hongqin Zhuang ${ }^{1,2+}$, Di Zhang ${ }^{1}$, Yao Wang ${ }^{1,3^{*}}$ and Zi-Chun Hua ${ }^{1,2^{*}}$
}

\begin{abstract}
Background: The urokinase-type plasminogen activator receptor (UPAR) is an important regulator of ECM proteolysis, cell-ECM interactions and cell signaling. UPAR and heat shock proteins HSP70 and MRJ (DNAJB6) have been implicated in tumor growth and metastasis. We have reported recently that MRJ (DNAJB6, a heat shock protein) can interact with UPAR and enhance cell adhesion. Here, we identified another heat shock protein HSP70 as a novel uPAR-interacting protein.
\end{abstract}

Methods: We performed co-immunoprecipitation in human embryonic kidney (HEK) 293 and colon cancer HCT116 cells as well as immunofluorence assays in HEK293 cells stably transfected with uPAR to investigate the association of suPAR with HSP70/MRJ. To understand the biological functions of the triple complex of suPAR/HSP70/MRJ, we determined whether HSP70 and/or MRJ regulated UPAR-mediated cell invasion, migration, adhesion to vitronectin and MAPK pathway in two pair of human tumor cells (UPAR negative HEK293 cells vs HEK293 cells stably transfected with UPAR and HCT116 cells stably transfected with antisense-uPAR vs HCT116 mock cells transfected with vector only) using transwell assay, wound healing assay, quantitative RT-PCR analyzing mmp2 and mmp9 transcription levels, cell adhesion assay and Western blotting assay.

Results: HSP70 and MRJ formed a triple complex with UPAR and over-expression of MRJ enhanced the interaction between HSP70 and UPAR, while knockdown of MRJ decreased soluble UPAR in HCT116 cells $(P<0.05)$ and reduced the formation of the triple complex, suggesting that MRJ may act as an UPAR-specific adaptor protein to link UPAR to HSP70. Further experiments showed that knockdown of HSP70 and/or MRJ by siRNA inhibited uPAR-mediated cell adhesion to vitronectin as well as suppressed cell invasion and migration. Knockdown of HSP70 and/or MRJ inhibited expression of invasion related genes mmp2 and mmp9. Finally, HSP70 and/or MRJ up-regulated phosphorylation levels of ERK1/2 and FAK suggesting MAPK pathway was involved. All the biological function experiments in cell level showed an additive effect when HSP70 and MRJ were regulated simultaneously indicating their collaborated regulation effects on UPAR.

Conclusions: These findings may offer a novel insight into the interactions between UPAR and HSP70/MRJ and their functions in cell adhesion and migration may provide more understanding of the roles in regulating cancer metastasis.

Keywords: Heat shock protein HSP70, MRJ, Association, Urokinase receptor, Cell adhesion, Cell migration

\footnotetext{
*Correspondence: yao_wang_au@yahoo.com; zchua@nju.edu.cn

${ }^{\dagger}$ Equal contributors

'The State Key Laboratory of Pharmaceutical Biotechnology, Nanjing University, Nanjing 210093, Jiangsu, P.R. China

${ }^{2}$ Changzhou High-Tech Research Institute of Nanjing University and Jiangsu

Target Pharma Laboratories Inc, Changzhou 213164, Jiangsu, P.R. China

Full list of author information is available at the end of the article
} 


\section{Background}

The urokinase-type plasminogen activator (uPA) receptor (UPAR) and its ligand UPA are involved in numerous physiological and pathological processes including pericellular proteolysis, wound healing, tissue regeneration and tumor progression [1-3]. The UPAR protein belongs to the Ly-6/uPAR/ $\alpha$-neurotoxin protein domain family [4] and is a single chain three-domain glycoprotein designated DI, DII and DIII [5]. Since UPAR is located on the cell surface by a glycosyl phosphatidylinositol (GPI) anchor and lacks a trans-membrane domain, it needs to interact with other partner proteins including integrins to activate cellular signaling pathways [6-8]. There also exist three soluble forms of UPAR, DI, DIDII and DIDIIDIII, which are present in cancer cells, urine, blood and cerebrospinal fluid [9-12]. UPAR expression is up-regulated during inflammation [13] and many other diseases [14] including cancer, and its expression levels correlate with poor prognosis [15-18]. uPA binds to uPAR and converts the zymogen plasminogen into plasmin which promotes degradation of ECM by direct digestion and activation of pro-matrix metalloproteases (MMPs), including MMP-2, $-9,-12$ and -13 [19]. In addition to the binding of UPA, uPAR initiates signal transduction pathways by interacting with other molecules such as vitronectin, integrins $\beta 1 / 2 / 3$, cytokeratin $8 / 18$ and EGFR (epidermal growth factor receptor) $[1,20]$. These interactions with uPAR result in various functional consequences depending on the specific interacting protein. For example, vitronectin binds to UPAR, and once phosphorylated, regulates uPAR-dependent cell adhesion $[8,21,22]$. However, to date, the numerous and varied roles of uPAR in cell adhesion, migration, proliferation, angiogenesis and cancer metastasis are not completely explained by identified known protein interactions. We therefore speculate that there are still additional and as yet unidentified uPAR partners. Recently we have described an uPAR binding protein, heat shock protein MRJ, which can regulate uPAR-mediated cell adhesion to vitronectin [23]. In this paper, we identified another heat shock protein HSP70 which can also interact with uPAR.

Heat shock proteins (HSPs) are a set of highly conserved proteins that are inducible by a wide variety of physiological and environmental factors including anti-cancer chemotherapy, thus allowing the cells to survive to lethal conditions. The HSP70 protein is a member of the DnaK/ HSP70 class (HSP701A1, 72KDa) (NM_005345). The MRJ protein is member 6 of the DnaJ/HSP40 homolog subfamily B (DnaJB6) (NM_005494). MRJ is an essential co-chaperone of HSP70, with the N-terminal J-domain necessary for its interaction with HSP70 and its chaperone activity $[24,25]$. MRJ drives the substrate specificity of HSP70. They usually cooperate in the binding of proteins at intermediate stages of folding, assembly, and translocation across membranes in almost all cellular compartments. Similar to uPAR, expression levels of HSP70 and MRJ are correlated with metastasis and poor prognosis in breast cancer cells [26]. To date, little is known about interaction of HSP70/MRJ complex with uPAR and the biological significance of HSP70/MRJ complex in regulation of UPAR and its signaling. To answer these questions, we show here that the heat shock proteins HSP70 and MRJ form a triple complex and interact functionally with uPAR to increase uPAR-mediated cell migration and adhesion to vitronectin.

\section{Methods}

\section{Materials}

The vector pRNAT-U6.1/neo was purchased from Genescript, USA. The Plasmid HSP70-PRK5 was kindly provided by Dr. Fei Dow from Beijing Normal University. The anti-ERK1/2 and anti-phospho-ERK1/2 antibodies were purchased from Cell Signaling Technology, antiFAK, anti-phospho-(pY397) FAK and anti-HSP70 from BD Biosciences, anti-phospho-(pS473) AKT from Epitomics, anti-uPAR, anti-HA, anti-MRJ, anti-flag, antitubulin, anti-GAPDH, anti- $\beta$-actin, goat-anti mouse HRP IgG and goat anti-rabbit HRP IgG antibodies from Santa Cruz, and anti-Alexa Fluor ${ }^{\mathrm{rt}}$-labeled secondary antibodies from Invitrogen.

\section{Cell culture and transfection}

The wild type human embryonic kidney (HEK) 293 and human colon carcinoma (HCT116) and human epithelial cervical cancer Hela cell lines were purchased from the American Type Culture Collection (ATCC, Philadelphia, PA, USA). The HEK293T (HEK 293-uPAR) cells stably transfected with uPAR (HEK 293-uPAR) were kindly provided by Dr. Ying Wei (University of California, San Francisco) [22]. The HCT116 cells stably transfected with antisense-uPAR and HCT116 mock cells (vectoronly transfected clone) were generated previously [27]. Cells were all grown in Dulbecco's modified Eagle's medium (DMEM) (Hyclone) supplemented with 10\% FBS (Hyclone) and $50 \mathrm{U} / \mathrm{ml}$ penicillin/streptomycin, and maintained in a humidified atmosphere containing $5 \% \mathrm{CO}_{2}$ at $37^{\circ} \mathrm{C}$. The stable transfected HEK293-uPAR cells, HCT116 mock cells and antisense-uPAR HCT116 cells were cultured in the presence of $0.9 \mathrm{mg} / \mathrm{ml}$ and $0.6 \mathrm{mg} / \mathrm{ml} \mathrm{G418} \mathrm{(Geneticin)} \mathrm{to} \mathrm{maintain} \mathrm{selection,} \mathrm{re-}$ spectively. Cell transfection was performed using Lipofectamine $^{\circ} 2000$ transfection reagent (Invitrogen).

\section{Expression vector construction}

The vectors encoding uPAR-HA (HA tag is influenza hem-agglutinin: YDYDVPDYA) and MRJ-flag (flag-tag is a peptide with eight amino acids: DYKDDDDK) were generated by amplifying the human UPAR cDNA and MRJ cDNA and then cloned into the PCI-HA and PRK5-flag 
vectors respectively [23]. The negative control of UPAR siRNA vector which has the same nucleotide composition but not the same sequence as the UPAR siRNA was cloned into PRNAT-U6.1/neo designated as psiSC-uPAR. The HSP70 siRNA vector (psiHSP70) was constructed previously in our laboratory [28]. The MRJ siRNA insert sequence was designed using siRNA designer software and siRNA Construct Builder software (Genscript). MRJ siRNA was designed and constructed into PRNATU6.1/neo using BamHI/HindIII (Takara) and designated as psiMRJ. A negative control for siRNA as a scrambled sequence of the MRJ siRNA target sequence was also cloned into PRNAT-U6.1/neo designated as psiSC.

\section{Real-time quantitative PCR}

Total RNA was isolated from tumor cells with the TRIzol reagent (Invitrogen, Rockville, MD) according to the manufacturer's instructions. $2 \mu \mathrm{g}$ of total RNA was subjected to reverse transcription using RevertAid ${ }^{\mathrm{Tm}}$ First-Strand cDNA Synthesis Kit (Fermentas, Lithuania) with random hexamer primer. $8 \mu \mathrm{l}$ of the cDNA solution (after 50-100 times dilution) was used for real-time PCR. The genes were amplified in a $20 \mu \mathrm{l}$ reaction using Power $\mathrm{SYBR}^{\circ}$ Green PCR Master Mix (Applied Biosystems by life technologies, UK) according to the manufacturer's protocol. The PCR primers were synthesized and shown as: mmp2 upstream: CCGTCGC CCATCATCAA; mmp2 downstream: GGTATTGCACT GCCAACTCTTTG; mmp9 upstream: GGACGATGCCT GCAAGT; mmp9 downstream: ACAAATACAGCTGGTT CCCAATC; upa upstream: GTGGATGTGCCCTGAA GGA; upa downstream: TGCGGATCCAGGGTAAGAAG; upar upstream: GAATGGCCGCCAGTGTTACAG; upar downstream: TGGGCATGTTGGCACATTG; mrj upstream: AAGTGCTGTCGGATGCTAAG; mrj downstream: CCTGAAGACATCATCTGGGT; hsp70 upstream: GGGCCTTTCCAAGATTGCTGT; hsp70 downstream: ATCTCTGCATGTAGAAACCGGAAA. The GAPDH (glyceraldehyde 3-phosphatase dehydrogenase) was used as the reference gene and relative mRNA levels were determined using the $2^{-\Delta \Delta C t}$ method. Three independent experiments were performed. Student $t$-test was performed when comparing tumor cells transfected with psiSC, psiMRJ, psiHSP70 and psiMRJ plus psiHSP70 groups. The differences between scramble groups psiSC, psiMRJ, psiHSP70, psiMRJ plus psiHSP70 were significant $(P<0.05)$.

\section{Co-Immunnoprecipitation (Co-IP) and Western blotting}

Transfected and control cells were harvested, washed twice with cold PBS and lysed in lysis buffer $(50 \mathrm{mM}$ Tris$\mathrm{HCl}$ (pH 7.4), $250 \mathrm{mM} \mathrm{NaCl}, 0.5 \%$ Triton-X 100, $50 \mathrm{mM}$ $\mathrm{NaF}, 2 \mathrm{mM}$ EDTA, $1 \mathrm{mM} \mathrm{Na} \mathrm{VO}_{4}$ and protein inhibitor cocktail) for $45 \mathrm{~min}$. For immunoprecipitation, the lysates were incubated with primary antibody overnight at $4^{\circ} \mathrm{C}$, and then with Protein $\mathrm{G}$ beads for 2 hours at $4^{\circ} \mathrm{C}$. After washing the Protein $G$ beads for 4 times with lysis buffer to eliminate the proteins in nonspecific adsorption, the proteins binding to the beads were subjected to WB analysis. For Western blotting, equal amounts of proteins were subjected to SDS-PAGE and the membrane was incubated for 1 hour in PBS with 0.1\% Tween-20 (PBST) containing 5\% non-fat skim milk for 1 hour at room temperature. Subsequently, the membrane was incubated for 1 hour at room temperature or $4{ }^{\circ} \mathrm{C}$ overnight with primary antibody in PBST containing 5\% non-fat milk, developed with HRP (horseradish peroxidase)-conjugated goat anti-mouse IgG (Santa Cruz Biotechnology, Santa Cruz, CA, U.S.A.) or anti-rabbit IgG and an $\mathrm{ECL}^{\bullet}$ (enhanced chemiluminescence) detection system (Amersham Biosciences, Arlington Heights, IL, U.S.A.), followed by exposure to X-ray film. For loading control, the membranes were stripped and probed with antibodies for GAPDH, $\alpha$ tubulin or $\beta$-actin, as per standard protocols. In addition, the HCT116 mock cells were transfected with selected amount of psiHSP70 or psiMRJ alone or in combination or with $10 \mu \mathrm{M}$ of the proteasome inhibitor, MG-132 (Sigma), for 8 hours in serum-free media. Cells were lysed and probed with uPAR, HSP70, or MRJ antibody as described above. The blots were also probed with $\beta$-actin antibody for comparison.

\section{Immunofluorescence microscopy}

Tumor cells were washed twice in PBS and fixed for 1 hour in cold PBS containing $4 \%$ formaldehyde. The cells were washed three times in cold PBS and permeabilized for an additional 1 hour in 0.5\% Triton X-100. Non-specific staining was blocked with $3 \%$ BSA. Primary antibodies were added for 1 hour at $37^{\circ} \mathrm{C}$. After washing with PBS, species specific Alexa Fluor ${ }^{\text {rix }}$-labeled secondary antibodies were added for 1 hour at $37^{\circ} \mathrm{C}$. After washing with PBS, cover slips were mounted on the glass slides using glycerin. Control experiments in the absence of primary antibodies were run in parallel using the same procedure. Fluorescence microscopy was performed by microscopy (Carl Zeiss Axioplan 2).

\section{Flow cytometry}

Cell-surface UPAR levels were measured by flow cytometry. Treated cells were harvested, washed and resuspended in cold PBS containing $1 \%$ BSA $\left(2 \times 10^{6}\right.$ cells $\left./ \mathrm{ml}\right)$. Treated tumor cells were labeled with anti-human uPAR monoclonal antibody for $30 \mathrm{~min}$ on ice in the dark and immediately analysed by flow cytometry in APC channel. Suitable negative isotype controls were used to rule out background fluorescence. The data were generated by cytofluorometric analyses of 10,000 events. All data were analysed using CELLQuest software (Becton-Dickinson). For cell apoptosis analysis, cells were transfected with 
different siRNA vectors. Twenty-four hours later, the ratio of cell apoptosis was determined by flow cytometry analysis as previously described [28].

\section{Cell cycle analysis}

Cells $\left(2 \times 10^{6}\right)$ were treated with psiHSP70 and psiMRJ alone or in combination for 24 hours. Cells were then harvested, washed in PBS, resuspended gently in $5 \mathrm{ml}$ of $100 \%$ ethanol, and fixed at $25^{\circ} \mathrm{C}$ for 1 hour. After washing with $\mathrm{PBS}$, cells were incubated with DNase-free RNase A $(200 \mu \mathrm{g} / \mathrm{ml})$ at $37^{\circ} \mathrm{C}$ for 1 hour and washed with PBS. PI $(10 \mu \mathrm{g} / \mathrm{ml})$ was added and the cells were incubated at $37^{\circ} \mathrm{C}$ for $5 \mathrm{~min}$. The distribution of cells with differing DNA content was analyzed on a FACSCalibur flow cytometer with CellQuest software (BD Biosciences, CA, USA) at an excitation wavelength of $530 \mathrm{~nm}$. Fluorescence emission was measured using a $620 \mathrm{~nm}$ band pass filter.

\section{Cell proliferation assay}

Cells in the exponential growth phase were seeded into a 96-well plate at a density of 5000 cells per well. After 24 hours, cells were transfected with psiHSP70 and/or psiMRJ. The cells were incubated at $37^{\circ} \mathrm{C}$ for 24 hours, then the cell viability was determined by the colorimetric MTT [3-(4, 5-dimethylthiazol-2-yl)-2, 5-diphenyl-2Htetrazolium bromide] assay at wave length $570 \mathrm{~nm}$ by TECAN Safire Fluorescence Absorbance and Luminescence Reader (Vienna, VA, USA). The cell viability was calculated according to the formula: Cell viability (\%) =

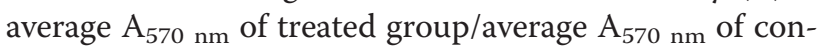
trol group $\times 100 \%$. Each experiment was performed in quadruplicate and repeated at least three times.

\section{F-actin staining assay}

After the indicated treatments, cells were fixed with $4 \%$ formaldehyde for 1 hour, penetrated with $0.5 \%$ Triton X-100 for 1 hour, and then stained with Texas Red-X phalloidin for 1 hour. F-actin was then visualized by microscopy (Carl Zeiss, Axioplan 2).

\section{Cell adhesion assay}

96-well plates were pre-coated with vitronectin $(1 \mu \mathrm{g} / \mathrm{ml})$, or fibronectin $(5 \mu \mathrm{g} / \mathrm{ml})$, or bovine serum albumin (BSA, $10 \mu \mathrm{g} / \mathrm{ml})$ at $37^{\circ} \mathrm{C}$ overnight, and the wells were blocked with $1 \% \mathrm{BSA}$ at $37^{\circ} \mathrm{C}$ for 1 hour. After washing with DMEM serum-free medium for three times, cells were blocked with $0.1 \%$ BSA in DMEM and added to coated plates $\left(5 \times 10^{3}-1 \times 10^{4}\right.$ cells $/ 100 \mu \mathrm{l}$ per well). After incubation at $37^{\circ} \mathrm{C}$ for 1 hour, the plates were washed with PBS for 1-3 times till no cells were left in the BSA coated wells. Adherent cells were fixed with methanol for $10 \mathrm{~min}$ at room temperature, stained with Giemsa and quantified by reading absorbance at $550 \mathrm{~nm}$ using Safire Fluorescence
Absorbance and Luminescence Reader (TECAN). All experiments were repeated at least three times.

\section{Wound-healing assay}

Transfected cells were plated in 12-well culture plates to form cell monolayer (near 70\% confluence). After serum starvation for 12 hours, a wound was made with a sterile P-200 micropipette to scrape off the cells. The wells were then washed three times with PBS to remove nonadherent cells and incubated in fresh medium containing FBS. The progress of wound closure was monitored with microphotographs of $\times 10$ magnification taken with light microscope (Carl Zeiss Axioplan 2) at the beginning and the end of the experiments after washing with PBS.

\section{Transwell assay}

To determine cell migration and invasion, transwell assay was carried out using a 24 well cell culture insert with $8 \mathrm{~mm}$ pore (3097, Falcon-Becton Dickinson, USA). Transfected cells were cultured in serum-free medium overnight. DMEM containing 20\% FBS was used as a chemotactic attractant in lower compartment of the Boyden chamber. Single-cell suspensions were plated at the concentration of $10^{5}$ cells $/ \mathrm{ml}$ in $0.5 \mathrm{ml}$ serum-free medium, 1\% BSA per well into upper chamber for 24 hours. Cells from the upper surface of the filter were removed with a cotton swab; those underneath were fixed with $4 \%$ paraformaldehyde prior to staining with $0.5 \%$ crystal violet. Images were captured by $10 \times$ objective lens. Invaded or migrated cells were expressed as the average number of migrated cells per microscopic field over four fields per assay in triplicate experiments.

\section{Statistical analysis}

All data are presented as means \pm SD (standard deviation) of at least three independent experiments, each performed at least in triplicate, when normally distributed. The statistical significance of differences was determined by student's two-tailed $t$-test in 2 groups and one way ANOVA in multiple groups. Statistical differences are presented at probability levels of $P<0.05$, and $P<$ 0.01 . All data were analyzed with SPSS 13.0 software.

\section{Results}

HSP70 was associated with UPAR and formation of the UPAR-HSP70 complex was regulated by MRJ and HSP70

We have reported that MRJ can interact with UPAR by a yeast two-hybrid screen, GST-pull down, co-IP and confocal microscopy analyses [23]. Since MRJ has the ability to function together with HSP70, we hypothesized that HSP70 might also interact with UPAR to form a triple complex. To this end, we first determined whether uPAR and HSP70 were associated in wild-type HCT116 cells that express high levels of uPAR. We prepared total 
lysates from the cells and carried out co-IP and Western blot analyses. As shown in Figure 1A, the HSP70 band was detected in the sample immunoprecipitated with anti-uPAR antibody, but was not detected in the respective IgG isotype controls, indicating a specific interaction between UPAR and HSP70.

To further confirm the interaction between HSP70 and UPAR, vectors expressing epitope tagged HSP70flag, uPAR-HA and MRJ-flag were generated. Co-IP assays were performed using extracts from HEK293T cells that had been co-transfected with different combinations of these vectors. As shown in Figure 1B, when cells cotransfected with uPAR-HA and HSP70-flag, the HSP70flag protein was detected in lysate immunoprecipitated with anti-HA (uPAR) antibody, and $35 \mathrm{kDa}$ uPAR-HA protein was detected after immunoprecipitated with antiflag (HSP70) antibody.

To determinate whether MRJ was involved in the uPAR/ HSP70 complex, HEK293T cells were co-transfected with uPAR-HA, or uPAR-HA plus HSP70-flag, or uPAR-HA plus HSP70-flag plus MRJ-flag and then precipitated with anti-HA antibody (Figure 1C). The MRJ-flag protein was
A

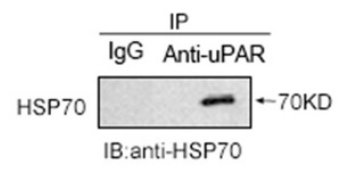

B

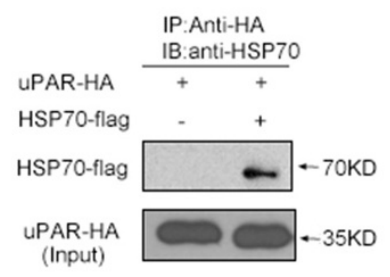

E

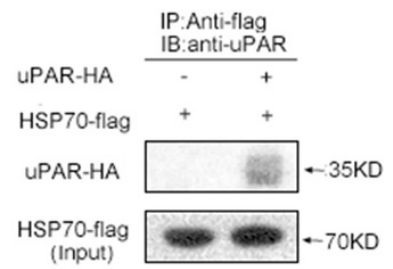

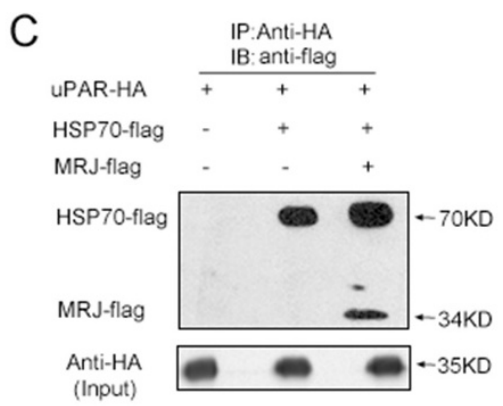
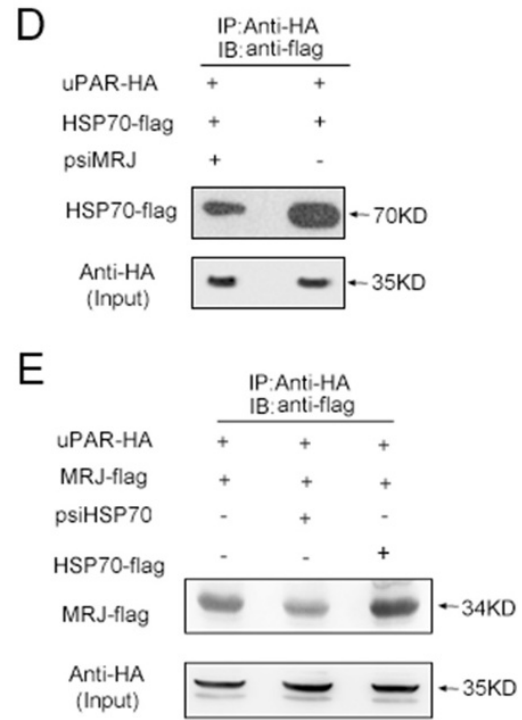

Figure 1 Association of uPAR with HSP70 and regulation of the uPAR/HSP70 complex by MRJ. (A) Co-IP of UPAR and HSP70 in HCT116 cells. Cell lysates were immunoprecipitated with anti-uPAR antibody and IgG and the Western blot was probed with anti-HSP70 antibody. (B) Co-IP of UPAR and HSP70 in HEK293 cells co-transfected with HSP70-flag and UPAR-HA constructs or transfected with uPAR-HA (top lanes) or HSP70-flag alone (down lanes). IP was performed using anti-HA or anti-flag antibody and WB was performed using anti-flag antibody (upper blot) or anti-HA antibody (lower blot). $6 \%$ or $2 \%$ of the amount of the original cell lysates used for IP was loaded as an input control and visualized with anti-HA antibody (top lanes) or anti-flag antibody (down lanes), respectively. (C) Over-expression of MRJ increased HSP70 in HEK293 cells. The cells were transfected with UPAR-HA, or UPAR-HA plus HSP70-flag or UPAR-HA plus HSP70-flag plus MRJ-flag. IP was performed using anti-HA antibody and WB was then performed using anti-flag to detect the indicated proteins. Equal amounts of protein in each cell lysate were blotted with anti-HA antibody as controls. (D) Knockdown of MRJ decreased the HSP70 in HEK293T cells. The cells were co-transfected with uPAR-HA and HSP70-flag, with or without psiMRJ. Co-IP was performed using anti-HA antibody and WB was performed using anti-flag to detect the indicated proteins. Equal amounts of protein in each cell lysate were blotted with anti-HA antibody as controls. (E) HSP70 regulated the interaction among HSP70 and MRJ and uPAR. The cells were co-transfected with UPAR-HA plus MRJ-flag, or uPAR-HA plus MRJ-flag plus HSP70-prk5, or uPAR-HA plus MRJ-flag plus psiHSP70. Co-IP was performed using anti-HA antibody and WB was performed using anti-flag to detect the indicated proteins. Equal amounts of protein in each cell lysate were blotted with anti-HA antibody as controls. 
detected in lysate immunoprecipitated with anti-HA (UPAR) antibody indicating that uPAR, HSP70 and MRJ formed a triple complex in HEK293T cells. Moreover, the presence of exogenous MRJ or HSP70 could enhance the interaction among HSP70 and MRJ and UPAR. In addition, we found that knockdown of MRJ or HSP70 by its siRNA significantly decreased the interaction in the triple complex in HEK293T cells (Figure 1D and E) suggesting that formation of the complex was regulated by MRJ and HSP70.

\section{RNAi-mediated down-regulation of HSP70/MRJ reduced uPAR protein level}

To confirm the triple complex and to study the regulation of the interaction between MRJ, HSP70 and UPAR, siRNA expressing plasmids targeting UPAR (psiuPAR), MRJ (psiMRJ) and HSP70 (psiHSP70) were constructed and the interference effects were shown in Figure 2A, resulting in $30-60 \%$ decreases in their protein levels. We then examined whether suppression of HSP70 and/or MRJ by their siRNA had any effects on protein level of UPAR in the wild type HCT116 cells. The cells were transfected with psiSC, or psiMRJ, or psiHSP70, or psiMRJ plus psiHSP70, and Western blot analyses were performed. As shown in Figure 2B, the expression of uPAR was significantly decreased in cells transfected with psiMRJ, or psiHSP70, or psiMRJ plus psiHSP70 when compared to their psiSC scramble controls $(P<0.05)$. Furthermore,
UPAR protein decreased about 20\%-30\% in HCT116 cells transfected with psiMRJ or psiHSP70, while had about $50 \%$ decrease in HCT116 cells transfected with psiMRJ plus psiHSP70 indicating their additive effects (Figure 2B).

To further characterize the functional interaction of UPAR with HSP70 and MRJ, HCT116 mock cells were transfected with selected amount of psiHSP70 or psiMRJ respectively. As shown in Figure 3, UPAR was shown to be degraded in a dose-dependent manner and this degradation could be rescued by combining the treatment with the proteasome inhibitor MG-132. No significant change in the level of $\beta$-actin was noted. These results demonstrate that UPAR is probably a client protein of the HSP70/ MRJ chaperone complex and knockdown of HSP70/MRJ prevents stabilization of client protein and leads to the degradation of the client protein by the proteasome.

\section{HSP70 co-localized with uPAR in the cytoplasm}

We next examined whether the association between UPAR and HSP70 resulted in co-localization of the two proteins using double immunofluorescence staining in the HEK 293-uPAR cells. As shown in Figure 4A, uPAR and HSP70 co-localized throughout the cytoplasm in exponentially growing HEK 293-uPAR cells. To determine the cytoplasm distribution of uPAR and HSP70, a fluorescent nucleistainDAPI that is only present in the nucleolus, was used together with the anti-uPAR and anti-HSP70 antibodies. As

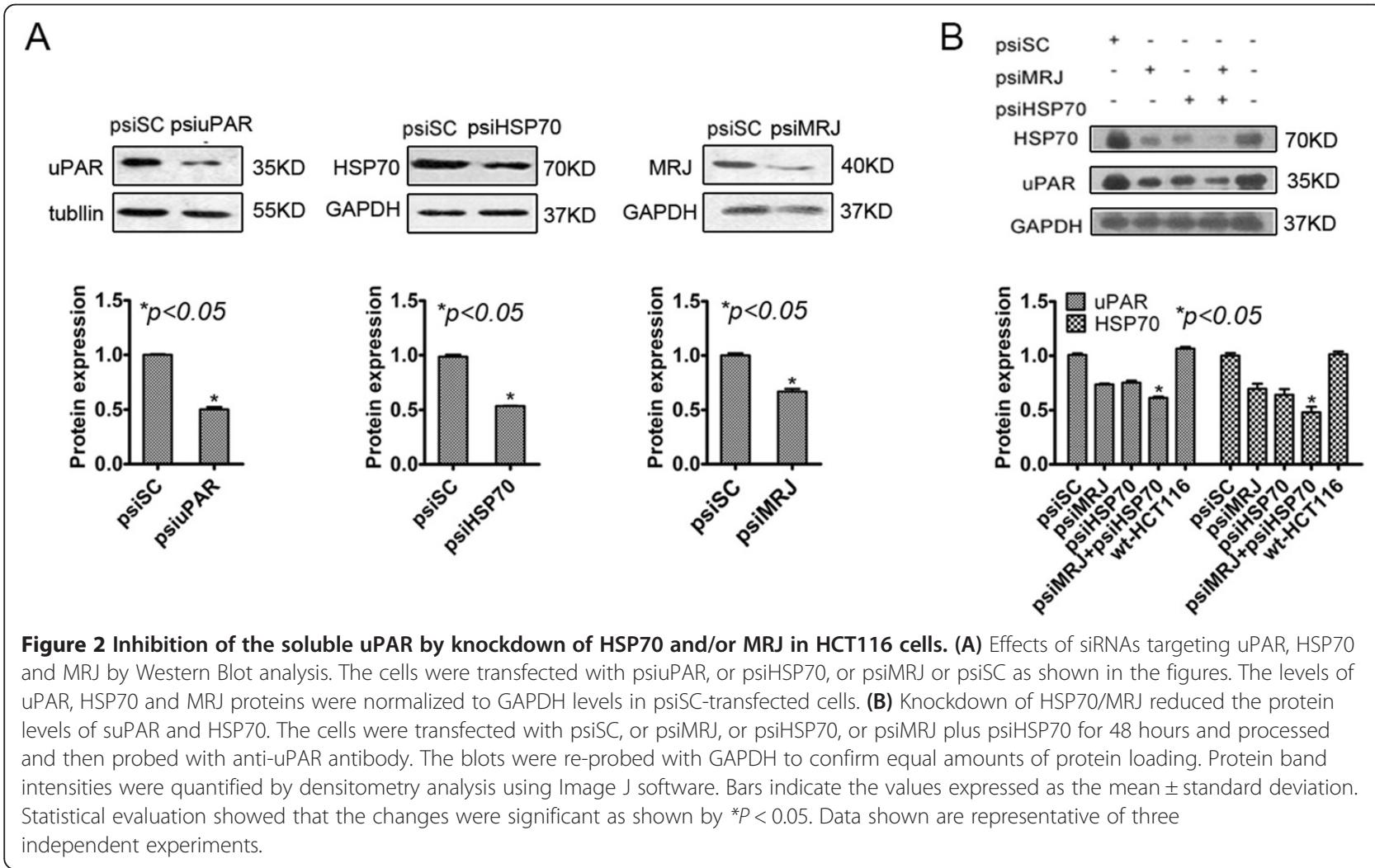




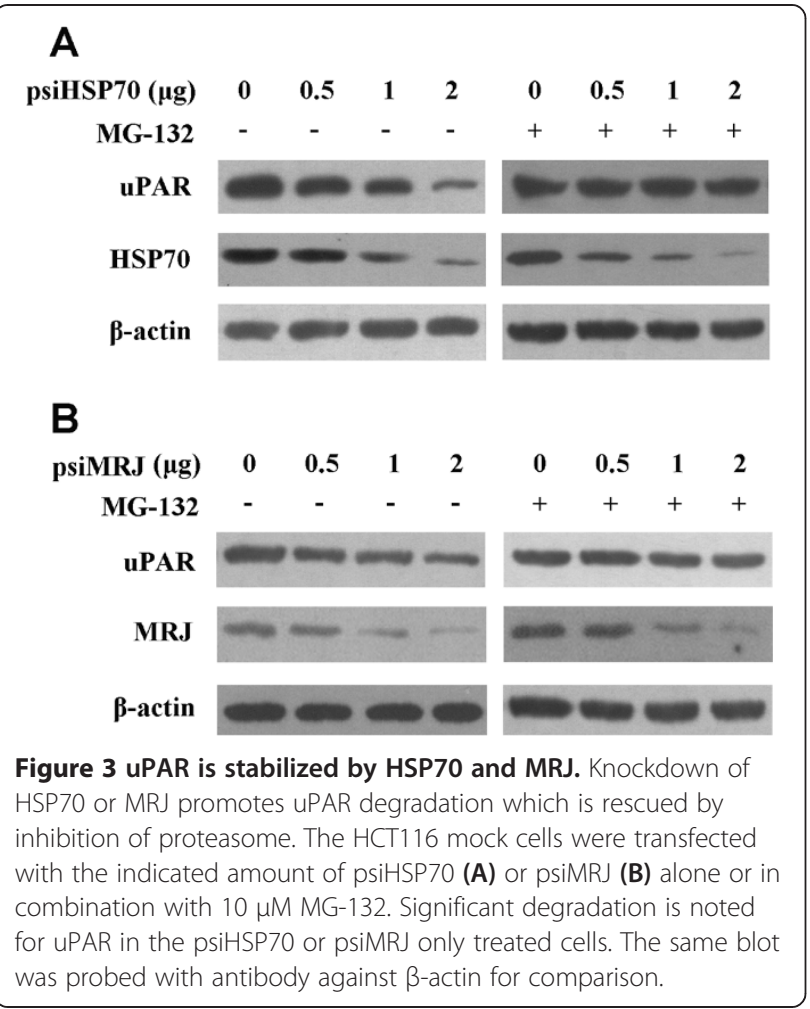

shown in Figure 4A, cells stained with secondary antibodies alone showed corresponding nuclear (DAPI) staining only (Blue) (up panel) suggesting the specificity of the indicated antibodies. In addition, we studied the intracellular distribution of soluble uPAR (His-tag suPAR) to determine whether HSP70 can also co-localize with suPAR. The data indicated that there was a significant overlap between exogenous suPAR and endogenous HSP70 in Hela cells as showed in Figure 4B. The association of suPAR and HSP70 was also observed in HEK293T and HEK 293uPAR cells (data not shown), as the extent of overlapping differed from cell to cell, suggesting the dynamic feature of the interaction between suPAR and HSP70. Collectively, our data demonstrated that UPAR co-localized with HSP70 in the cytoplasm.

\section{Knockdown of HSP70 and MRJ inhibited uPAR-mediated cell adhesion in HEK 293-uPAR cells}

To test the biological significance of the uPAR-MRJHSP70 interaction, we next examined whether HSP70 and/or MRJ regulated uPAR-dependent adhesion to the ligand vitronectin in cells. We have previously reported that MRJ regulates uPAR-dependent adhesion to vitronectin and UPAR is involved in adhesion of HEK293 cells stably transfected with uPAR to vitronectin [23]. As shown in Figure 5A, psiMRJ or psiHSP70 led to a 30\% reduction and $10 \%$ reduction respectively in HEK 293uPAR cells adhesion to vitronectin, while psiHSP70 and
psiMRJ in combination caused a 50\% reduction. However, all these siRNA plasmids alone or in combination had no obvious effect in adhesion of uPAR negative HEK293T cells to vitronectin, suggesting that the inhibition effects by knockdown of HSP70 and/or MRJ were mediated by uPAR. To confirm this, flow cytometry was performed to determine the cell surface uPAR level after knockdown of HSP70 and/or MRJ. As shown in Figure 5B, suppression of MRJ and HSP70 simultaneously exhibited significant decrease cell surface uPAR, while knockdown of HSP70 or MRJ alone had only weak inhibition for cell surface UPAR. The decreased cell surface uPAR by knockdown of MRJ and/or HSP70 may account for reduced cell adhesion and further confirmed the interaction between uPAR, HSP70 and MRJ.

\section{HSP70 and MRJ siRNAs suppressed cell invasion and migration}

Previous reports have verified the essential roles of UPAR in tumor invasion and metastasis [29,30]. To determine the role of HSP70/MRJ in cell invasion and migration, we conducted transwell and wound healing assays. In these experiments we used a pair of cell lines, HCT116 mock cells transfected with vector only that expresses high levels of UPAR and antisense-uPAR HCT116 cells that expresses low levels of uPAR. Transwell assay (Figure 6A) showed that cells transfected with psiHSP70 and psiMRJ alone exhibited a mild inhibition in the cell invasiveness, and combination of psiHSP70 and psiMRJ led to enhanced inhibition in HCT116 mock cells. However, no migration changes were evident in the antisense-uPAR HCT116 cells transfected with psiHSP70 and/or psiMRJ.

Wound healing scratch assay was also performed to examine whether down-regulation of HSP70 and MRJ could inhibit HCT116 cell migration. As shown in Figure 6B, 48 hours after the scratch, HCT116 mock cells transfected with psiSC migrated into and largely covered the original wound area, whereas those transfected with psiMRJ and/or psiHSP70 failed to cover a substantial portion of the wound. The results revealed that psiMRJ and psiHSP70 in combination led to enhanced inhibition of cell migration as compared to psiMRJ or psiHSP70 alone. However, the suppression of migration in the antisense-uPAR HCT116cells was not as significant as in HCT116 mock cells suggesting that the HSP70/MRJ suppressed migration at least partially through the inhibition of uPAR. Moreover, the cytoskeleton protein F-actin in HCT116 mock cells transfected with psiHSP70 or psiMRJ were redistributed when compared to control cells transfected with psiSC (Additional file 1). Taken together, these data suggest strongly that the interaction between HSP70/MRJ and UPAR play a role in regulating the migration of HCT116 cells in vitro. Furthermore we found that siRNA-mediated 


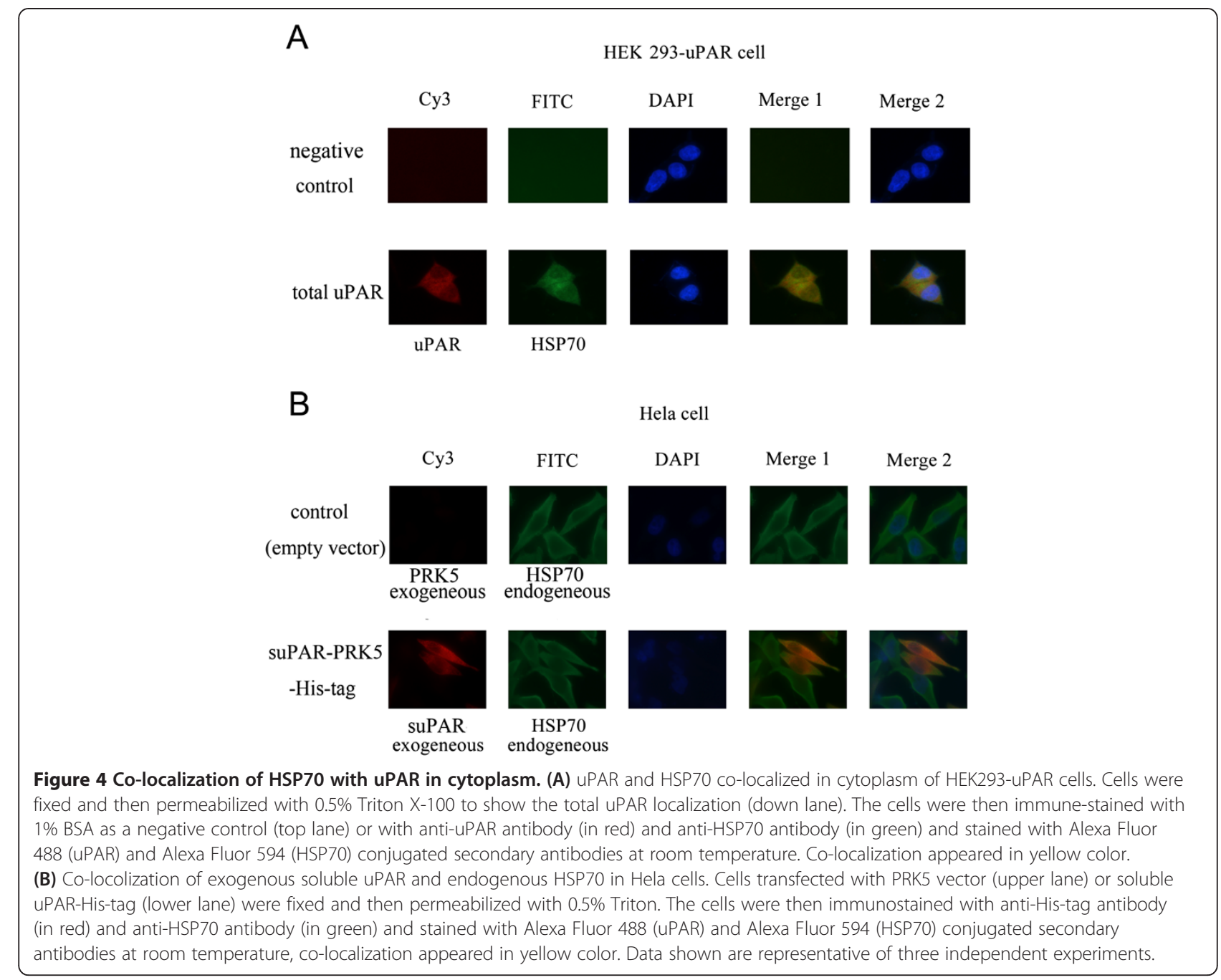

knockdown of HSP70 or/and MRJ had no obvious effects on cell proliferation and cell cycle in above cells (Additional file 2).

\section{Knockdown of HSP70/MRJ reduced expression of invasion} related genes $\mathrm{mmp2}$ and $\mathrm{mmp} 9$

It is documented that the uPAR initiates ECM enzyme degradation to promote extracellular proteolysis $[1,3]$. The UPA/uPAR system induces MMP activity and promotes cancer cell invasion and metastasis. Previous reports suggested that down-regulation of uPAR decreased the expression of mmp2 and mmp9 [31], which was consistent with our Q-PCR results as shown in Figure 7A. The levels of mmp 2 and mmp9 mRNAs were reduced about $50 \%$ and $90 \%$ respectively in HEK 293-uPAR cells transfected with psiuPAR (Figure 7A, left lane). To study whether inhibition of uPAR reduces expression of mmp2 and mmp9 genes, a pair of cell lines used in this paper were used (Figure 7A, right lane). In HEK 293-uPAR cells which express high amounts of uPAR, mmp2 and mmp9
mRNAs were over-expressed, while in HEK293T cells, mmp2 and mmp9 mRNAs were less-expressed. To study whether knockdown of HSP70/MRJ reduces expression of mmp2 and mmp9 genes, the HEK 293-uPAR cells were transfected with psiSC, or psiMRJ, or psiHSP70, or psiHSP70 plus psiMRJ. The HEK293T cells transfected with the above siRNAs were served as controls. As shown in Figure 7B, down-regulation of both HSP70 and MRJ expression in HEK 293-uPAR cells (Figure 7B, middle lane) caused more significant reduction of mmp2 and mmp9 mRNAs compared with that in HEK293T cells (Figure 7B, left lane). To eliminate the background and to determine effect of uPAR, comprehensive inhibition ratios were calculated with the results of the gene expression abundances in HEK 293-uPAR cells divided by the abundance in HEK293T cells (Figure 7B, right lane). The results suggested that the inhibition effects were mainly via UPAR. Collectively, these findings indicated that the effects of HSP70/MRJ on mmp2 and mmp9 gene expression in HEK 293-uPAR/HEK293T cells may be mediated by uPAR 


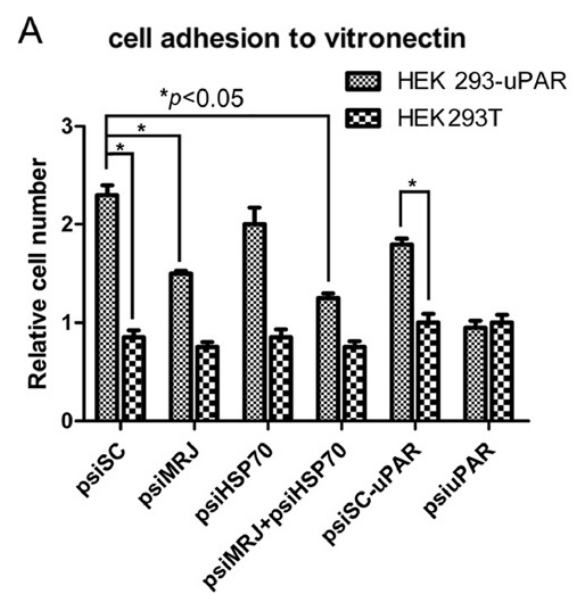

\section{B HEK 293-UPAR cell surface UPAR}

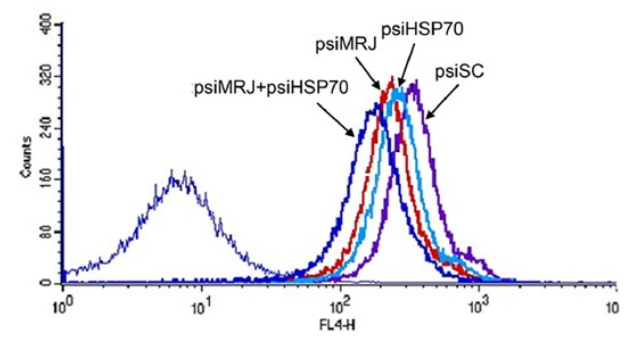

Figure 5 Decrease of uPAR-mediated adhesion to vitronectin by knockdown of MRJ and/or HSP70 in HEK293-uPAR and HEK293T cells. (A) Adhesion assay. The cells were transfected with psiSC, or psiMRJ, or psiHSP70 or psiMRJ plus psiHSP70 and grown on 96-well plates pre-coated with vitronectin $(1 \mathrm{\mu g} / \mathrm{ml})$ and stained with Giemsa and then quantified using Safire Fluorescence Absorbance and Luminescence Reader (TECAN). * $P<0.05$ : significantly different from the control. The experiments were repeated twice and performed in triplicate. (B) Reduction of uPAR on the cell surface by knockdown of MRJ/HSP70 using flow cytometry in HEK293-uPAR cells. The cells were transfected with psiSC, or psiMRJ, or psiHSP70, or psiMRJ plus psiHSP70. Forty-eight hours after transfection, cells were harvested and labeled with anti-human uPAR monoclonal antibody for $30 \mathrm{~min}$. After labeling, the cells were immediately analysed by flow cytometry in APC channel. Suitable negative isotype controls were used to rule out the background fluorescence.

through uPAR/HSP70/MRJ interactions. Moreover, these data further indirectly confirm that the association of uPAR/HSP70/MRJ plays a critical role in cell migration and invasion.

\section{Over-expression of HSP70 and MRJ increased phosphorylation of FAK, ERK1/2and AKT}

Binding of uPA to UPAR on the cell surface activates FAK, c-Src, H-Ras, AKT [32], extracellular signal-regulated kinase (ERK)/MAPK [33] and myosin light chain kinase [34]. UPAR binds directly to vitronectin and promotes activation of Rac1 [35]. These uPAR-dependent cell signaling events impact cell migration and survival. To determinate the effect of interaction between HSP70/MRJ and UPAR on uPAR-mediated intracellular signaling, we examined the signal proteins in cells over-expressing HSP70 and/or MRJ. The results indicated that in HCT116 mock cells, HSP70 or MRJ increased phosphorylation of FAK, ERK1/2 and AKT significantly $(P<0.05)$, while total FAK and ERK1/2 levels remained unchanged (Figure 8A, left lane). In contrast, in antisense-uPAR HCT116 cells, HSP70 or MRJ had no effect (Figure 8A, right lane), suggesting that HSP70 or MRJ activated these signal pathways through the interaction with UPAR. These data reinforce the association of HSP70/MRJ with UPAR and may explain mechanisms involved in the UPAR/HSP70/MRJ complexmediated cell adhesion, invasion and migration [36].

\section{Discussion}

Although uPAR and HSP70/MRJ are separately known to be involved in cell metabolism, physical, functional interaction of them resulting in changes of cell function have not been reported previously. The MRJ has been identified as an interacting protein of UPAR in our previous report. In this study, we report for the first time that HSP70, MRJ and UPAR form a triple complex and interact with one another, resulting in enhanced UPAR-dependent invasion, migration and adhesion to vitronectin.

The UPAR is an important regulator of several cellular processes including proliferation, migration, adhesion, as well as cell signaling. Similar to UPAR, over-expression of the HSP70 and MRJ increases tumor growth, cancer cell migration and metastatic potential. Recent findings indicate that cancer cells express high levels of HSPs which are closely correlated with poor prognosis [26]. MRJ is an essential co-chaperone of HSP70, with the Nterminal J-domain necessary for its interaction with HSP70 and its chaperone activity [24,25]. In breast cancer, similar to uPAR, HSP70 expression is correlated with metastasis and poor prognosis $[25,26]$. Under nonstress conditions, the HSP70 and MRJ have multiple housekeeping functions including folding and translocating newly synthesized proteins, as well as activating signaling molecules.

In our study UPAR was found to bind specifically to HSP70 but not HSP27 in HCT116 cells using co-IP analysis (data not shown). The HSP27 is a small heat shock protein which expresses high levels in many types of cancer cells [37]. Similarly the uPAR specifically bound to MRJ (DNAJB6), but not to DNAJB4 (another MRJ family member) in a breast cancer cell line MDA-MB231 by using co-IP assay. These data suggest that not all 


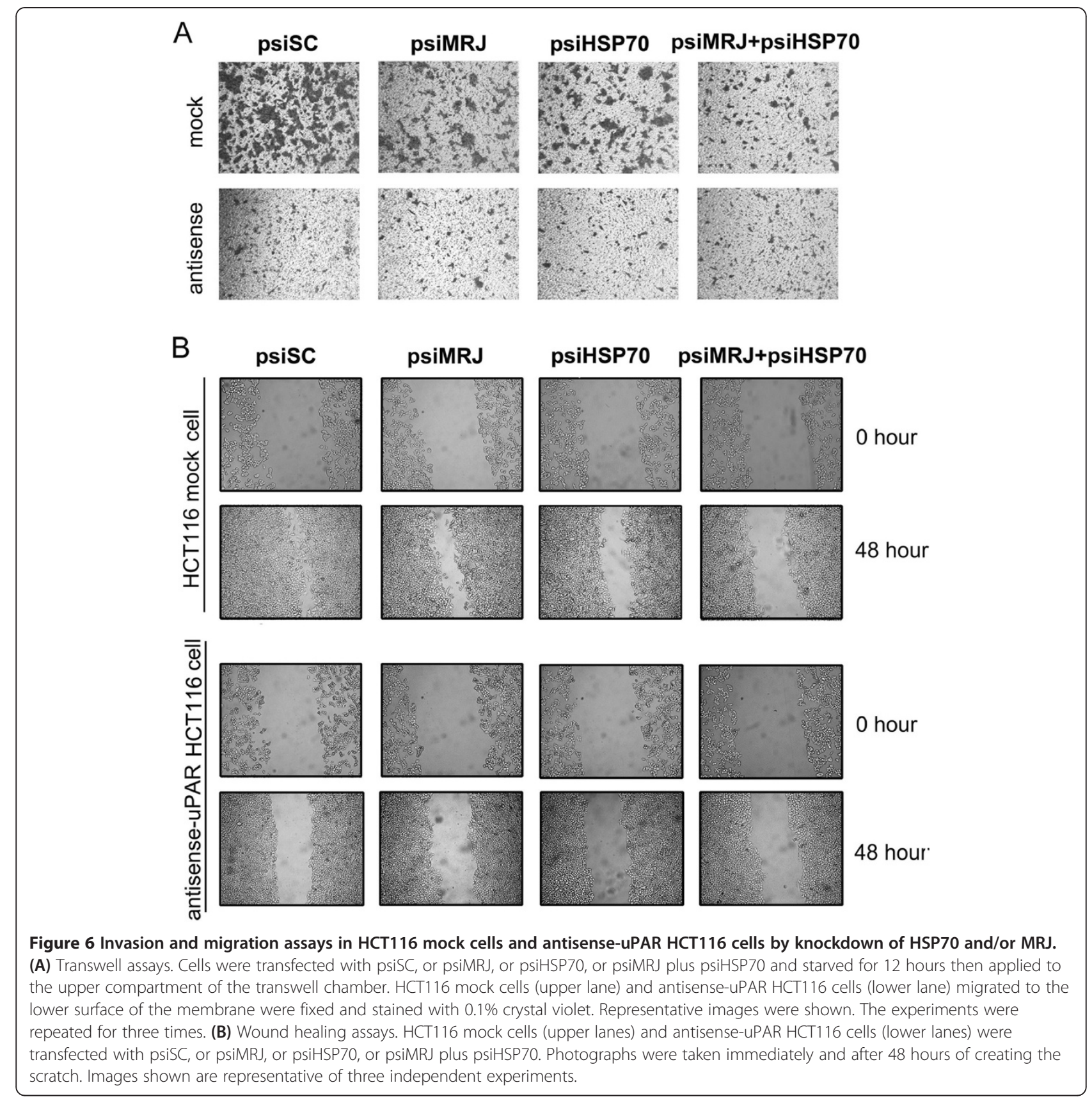

heat shock proteins can interact with UPAR and the interactions between UPAR and HSP70/MRJ are specific. These experiments were just additional controls to the standard IgG controls to determine specificity of the interaction between UPAR and HSP70 and MRJ. The coIPs of UPAR and HSP70/MRJ in wild-type HCT116 cells indicate that these proteins interact with each other when they expressed endogenously.

Our studies found that over-expression of MRJ or HSP70 enhanced the interaction between HSP70 and MRJ and UPAR, however knockdown of MRJ or HSP70 reduced the formation of the triple complex. The fact that
MRJ regulates the formation of uPAR-HSP70 complex suggests that MRJ may act as an UPAR-specific adaptor to link UPAR to the HSP70 protein. Furthermore, knockdown of HSP70 and/or MRJ also decreased cell-cytosol UPAR protein level in HCT116 cells and cell-surface UPAR protein level in HEK 293-uPAR cells. Further study suggested that UPAR may be a possible client protein of HSP70 and MRJ, and knockdown of HSP70 or MRJ may lead to the degradation of the client protein by the proteasome. Previous reports showed that when the uPAR/ uPA/PAI-1 complex associates with $\alpha 2 \mathrm{MR}-\mathrm{LRP}$ the entire complex is redistributed to clathrin coated vesicles for 


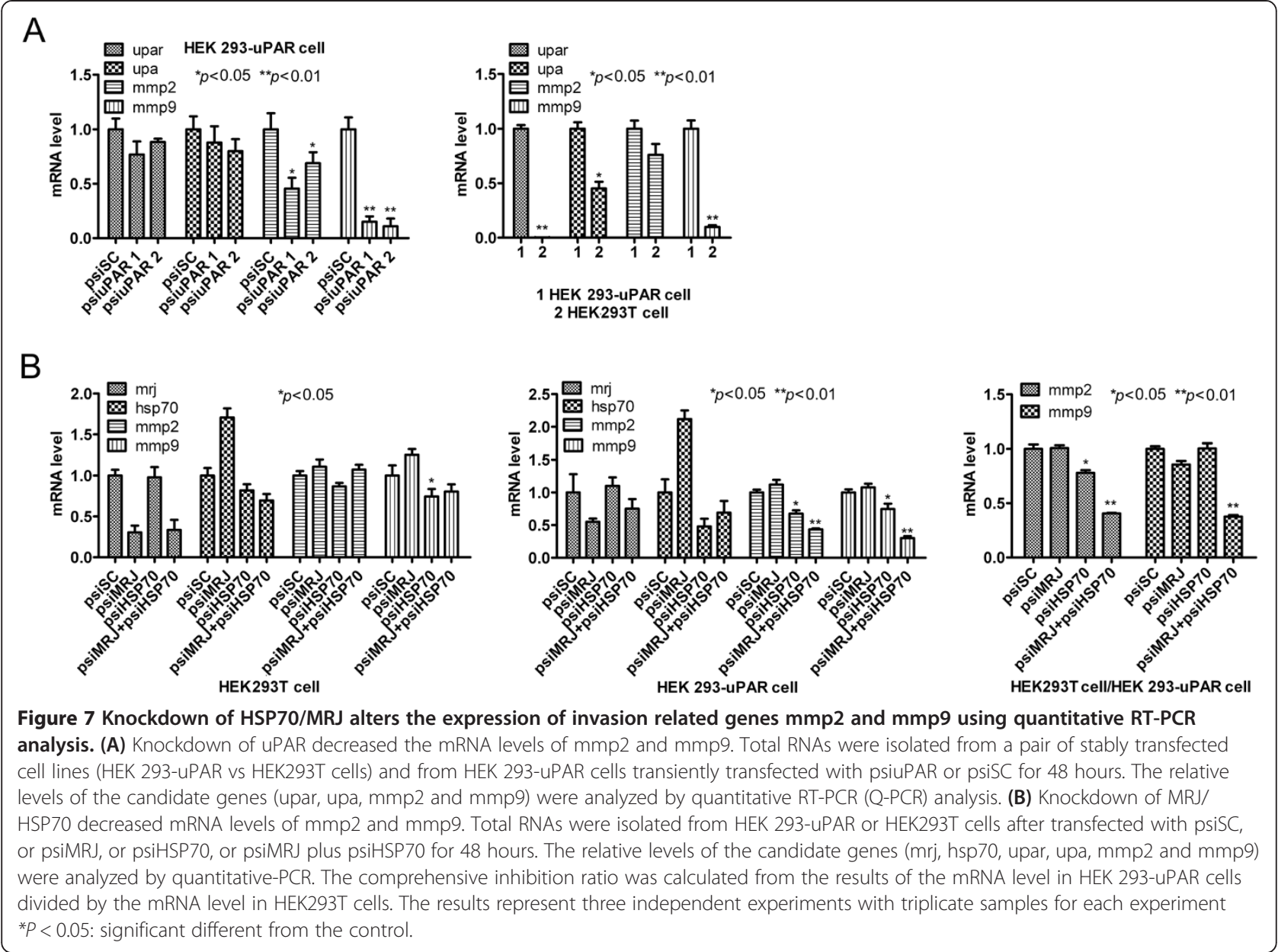

internalization, after which uPA and PAI-1 are degraded in the lysosome [38-40], but the internalized UPAR is recycled to the cell surface from the cytosol. MRJ is expressed predominantly in the cytoplasm, but HSP70 can interact with lipids and transport to cell membrane [41]. We thus speculate that HSP70 and MRJ form a complex and interact with uPAR in the cytoplasm and then HSP70 helps to recycle uPAR to the cell surface. It is still not clear whether the interaction between HSP70, MRJ and UPAR is taking place during uPAR folding, protein maturation and transportation to the cell surface and/or occurs after uPAR/uPA/PAI-1 internalization and uPAR recycling.

As shown in Figure 5A, the important uPAR/vitronectin interaction is at least in part regulated by HSP70 and/or MRJ. uPAR-dependent cell adhesion to the ECM protein vitronectin is an important event in wound healing, tissue remodeling, immune response and cancer development. Previous reports showed that on the surface of endothelial and U937 cells, uPAR can mediate cell adhesion to vitronectin $[42,43]$. The interaction between vitronectin and uPAR has also been implicated in regulating processes necessary for endothelial cell invasion and migration at vitronectin-rich extracellular matrix sites as well as facilitating intracellular signaling [42]. Functionally the interaction between UPAR and vitronectin can promote both cellular adhesion and migration [42-44] and may direct uPAR to focal contacts [8,45]. In HEK 293-uPAR cells, suppression of HSP70 or/and MRJ reduced uPAR-mediated cell adhesion to vitronectin significantly. We assume that MRJ and/or HSP70 knockdown may decrease cell-surface UPAR resulting in less interaction between UPAR and vitronectin. However, the exact mechanism how MRJ/HSP70 regulates uPARmediated cell adhesion to vitronectin is still not clear and need more study.

It is known that migrating cells must break down the established cell adhesion sites to detach from its substrate and establish new contact points between a cell and its underlying substrate to provide the necessary adhesion that a cell needs to move forward. Therefore, regulation of HSP70/MRJ on cell adhesion may play important roles in cell motility. As shown in this paper knockdown of HSP70 and/or MRJ inhibited cell migration by using wound healing and transwell assays in 


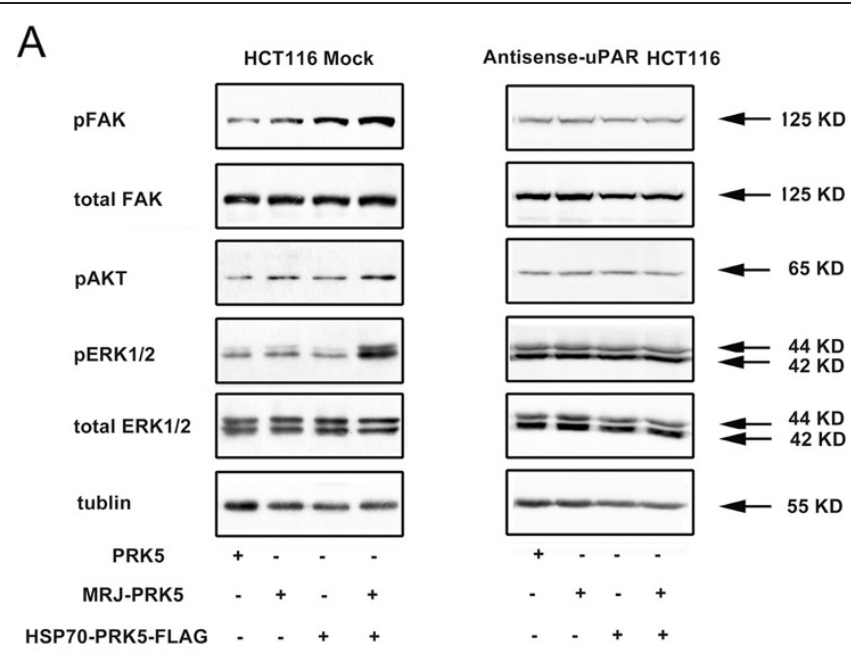

B
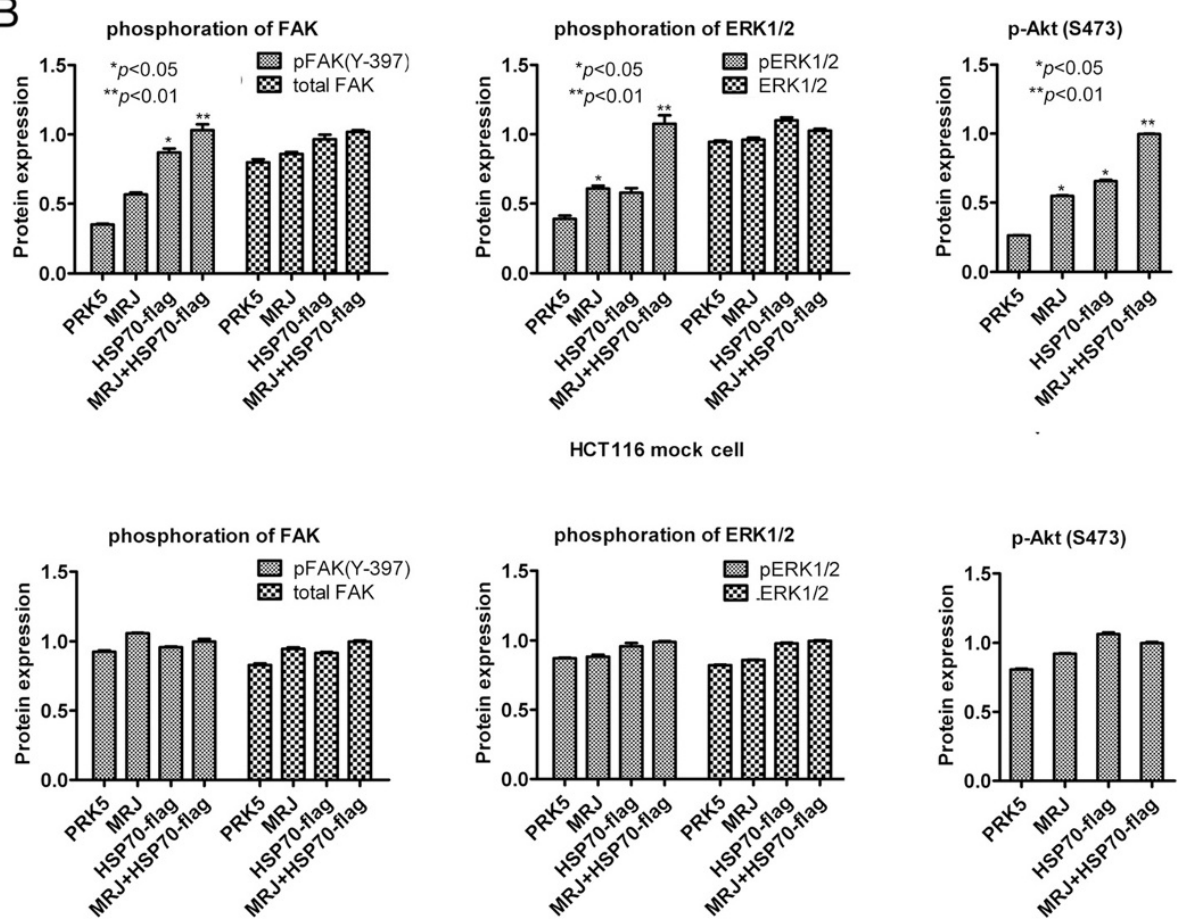

Figure 8 Phosphorylation of ERK1/2, FAK and AKT by over-expression of HSP70/MRJ is mediated by uPAR in HCT116 cells. (A) Cells were transfected with PRK5, or MRJ-PRK5, or HSP70-PRK5-flag or MRJ-PRK5 plus HSP70-PRK5-flag as shown in the figure. Forty-eight hours after transfection, cells were lysed and $60 \mu \mathrm{g}$ of total protein from each sample was loaded on SDS-PAGE. Then, the protein levels of ERK1/2, p-ERK1/2, FAK, p-FAK and p-AKT were analyzed by Western blot. Tubulin was used as a loading control. The results shown are representative of three independent experiments. (B) The bands shown in (A) were scanned by densitometry using Image J software. The ratios of phopho-ERK vs ERK, phospho-FAK vS FAK and P-AKT were calculated. The ratios in MRJ plus HSP70-transfected cells were arbitrarily taken as 1.0 and values of all others relative to it were calculated accordingly. The histagram shows the mean $\pm S D$, ${ }^{*} P<0.05$ : significantly different from the control, ${ }^{*} P<0.01$ : highly significantly different from the control.

HCT116 cells. Further experiments indicated that knockdown of HSP70 or MRJ had an effect on cytoskeleton reorganization. Recent studies have shown that the HSP70 can participate in cell migration by helping to target key regulatory proteins such as tissue transglutaminase to the leading edges of migrating cells [46]. Our results thus indicated that the interactions between HSP70, MRJ and UPAR can promote cell invasion and migration.

UPA-uPAR binding promotes cleaving plasminogen to plasmin which cleaves and activates matrix metalloproteases (MMPs) to enhance cell invasion, suggesting a mechanism that reducing cell-surface uPAR will decrease the expression of $\mathrm{mmp} 2$ and $\mathrm{mmp} 9$, resulting in 
total abrogation of matrix degradation. It is reported that inhibition of extracellular HSP70 and HSP90 reduce MMP2 activation and decrease cancer cell migration and invasion $[47,48]$. This and our data demonstrate that UPAR and HSP70/MRJ complex may promote matrix degradation, as knockdown of HSP70 and/or MRJ decreased the mRNA levels of mmp2 and mmp9, two important cancer invasion related genes.

Furthermore, cell signaling pathways, such as MEK1/ 2-ERK1/2 and PI3K-AKT, are shown to be downstream responses of UPAR activation. In this paper, we demonstrated that over-expression of HSP70 and/or MRJ enhanced phosphorylated signals of ERK, JNK, and AKT. Activation of these signaling pathways was correlated with significant increases in migration and invasion capacities when HSP70 and/or MRJ were over-expressed. Thus, we favor a model in which HSP70 or MRJinduced UPAR expression activates cell signaling pathways that are complementary in inducing the full spectrum of cellular changes observed in adhesion and migration [48].

\section{Conclusion}

In conclusion, our findings suggest a novel function of HSP70/MRJ/uPAR complex in cell adhesion, invasion and migration, and may provide more understanding in the mechanisms of uPAR-mediated cancer metastasis. The finding of triple complex interaction and its biological significance may promote basic research and further highlight the UPAR as a molecular target for therapy.

\section{Additional files}

\section{Additional file 1: Knockdown of HSP70/MRJ modulates cytoskeleton} reorganization. HCT116 mock cells were transfected with psiHSP70 or psiMRJ. F-actin was visualized with Texas Red-X phalloidin. Scale bar $=20 \mu \mathrm{m}$

Additional file 2: Knockdown of HSP70/MRJ has no obvious effects on cell proliferation and cell cycle. (A) HCT116 mock cells and antisense-UPAR HCT116 cells were transfected with psiHSP70 and/or psiMRJ. Twenty-four hours later, all cells were harvested for flow cytometry analysis. The experiments were carried out independently in triplicate. (B) HCT116 mock cells and antisense-uPAR HCT116 cells were transfected with psiHSP70 and/or psiMRJ, and the cell viability was assessed by MTT assay. (C) MCF-7 and MB-MDA-231 cells were transfected with psiHSP70 and/or MRJ. Twenty-four hours later, cells were fixed and stained with PI for flow cytometry as described in Materials and Methods. DNA histograms were modeled with CellQuest analysis software. Phase percentages for G0/ G1, S, and G2/M are depicted by bar graph. Data represent mean values of triplicate samples.

\section{Abbreviations \\ ECM: Extra cellular matrix; GPI: Glycosyl phosphatidylinositol; HSP: Heat shock protein; MRJ: Mammalian relative of DnaJ; PBS: Phosphate-buffered saline; SDS: Sodium dodecyl sulfate; uPA: Urokinase-type plasminogen activator; UPAR: UPA receptor.}

\section{Competing interests}

The authors declare no conflicts of interest with any other person or units.

\section{Authors' contributions}

All the authors have made substantial contributions to this work. YLL conducted study design and data collection and analysis; NNP and DZ performed data collection and analysis as well as drafted manuscript; HQZ contributed to study design, data collection and analysis and manuscript writing. As corresponding author, ZCH and YW designed and coordinated this research and provided guidance throughout this process. All authors read and approved the final manuscript.

\section{Acknowledgment}

The authors are grateful to grants from the Chinese National Natural Sciences Foundation (31200572 and 81121062), the Ministry of Science and Technology (2014CB744501, 2012CB967004), the Jiangsu Provincial Nature Science Foundation (BK2011228, BK2011573, BK2011048, BE20130630 and BZ2012050), the Bureau of Science and Technology of Changzhou, Jiangsu, China (CZ20120004, CM20122003, CZ20130011 and CE20135013).

\section{Author details}

${ }^{1}$ The State Key Laboratory of Pharmaceutical Biotechnology, Nanjing University, Nanjing 210093, Jiangsu, P.R. China. ${ }^{2}$ Changzhou High-Tech Research Institute of Nanjing University and Jiangsu Target Pharma Laboratories Inc, Changzhou 213164, Jiangsu, P.R. China. ${ }^{3}$ Division of Critical Care and Surgery, St. George Hospital, University of New South Wales, Sydney NSW2217, Australia.

Received: 19 August 2013 Accepted: 21 August 2014

Published: 30 August 2014

\section{References}

1. Blasi F, Carmeliet P: UPAR: a versatile signalling orchestrator. Nat Rev Mol Cell Biol 2002, 3(12):932-943.

2. Cohen RL, Xi XP, Crowley CW, Lucas BK, Levinson AD, Shuman MA: Effects of urokinase receptor occupancy on plasmin generation and proteolysis of basement membrane by human tumor cells. Blood 1991, 78(2):479-487.

3. Zhou HM, Nichols A, Meda P, Vassalli JD: Urokinase-type plasminogen activator and its receptor synergize to promote pathogenic proteolysis. EMBO J 2000, 19(17):4817-4826.

4. Wang YJDL, Johnson K, Selhamer JJ, Doe WF: Structure of the human urokinase receptor geneand its similarity to CD59 and the Ly- 6 family. Eur J Biochem 1994, 227(94 1175/1):6.

5. Ploug $M$, Ellis V: Structure-function relationships in the receptor for urokinase-type plasminogen activator. Comparison to other members of the Ly- 6 family and snake venom alpha-neurotoxins. FEBS Lett 1994 349(2):163-168.

6. Smith HW, Marshall CJ: Regulation of cell signalling by uPAR. Nat Rev Mol Cell Biol 2010, 11(1):23-36.

7. Ossowski L, Aguirre-Ghiso JA: Urokinase receptor and integrin partnership: coordination of signaling for cell adhesion, migration and growth. Curr Opin Cell Biol 2000, 12(5):613-620.

8. Madsen CD, Sidenius N: The interaction between urokinase receptor and vitronectin in cell adhesion and signalling. Eur J Cell Biol 2008, 87(8-9):617-629.

9. Thurison T, Lomholt AF, Rasch MG, Lund IK, Nielsen HJ, Christensen IJ, Hoyer-Hansen G: A new assay for measurement of the liberated domain I of the urokinase receptor in plasma improves the prediction of survival in colorectal cancer. Clin Chem 2010, 56(10):1636-1640.

10. Mustjoki S, Sidenius N, Sier CF, Blasi F, Elonen E, Alitalo R, Vaheri A: Soluble urokinase receptor levels correlate with number of circulating tumor cells in acute myeloid leukemia and decrease rapidly during chemotherapy. Cancer Res 2000, 60(24):7126-7132.

11. Chavakis T, Kanse SM, Yutzy B, Lijnen HR, Preissner KT: Vitronectin concentrates proteolytic activity on the cell surface and extracellular matrix by trapping soluble urokinase receptor-urokinase complexes. Blood 1998, 91(7):2305-2312.

12. Ploug M, Eriksen J, Plesner T, Hansen NE, Dano K: A soluble form of the glycolipid-anchored receptor for urokinase-type plasminogen activator is secreted from peripheral blood leukocytes from patients with paroxysmal nocturnal hemoglobinuria. Eur J Biochem 1992, 208(2):397-404.

13. Connolly BM, Choi EY, Gardsvoll H, Bey AL, Currie BM, Chavakis T, Liu S, Molinolo A, Ploug M, Leppla SH, Buqqe TH: Selective abrogation of the UPA-UPAR interaction in vivo reveals a novel role in suppression of fibrin-associated inflammation. Blood 2010, 116(9):1593-1603. 
14. Wei C, Moller CC, Altintas MM, Li J, Schwarz K, Zacchigna S, Xie L, Henger A, Schmid H, Rastaldi MP, Cowan P, Kretzler M, Parrilla R, Bendayan M, Gupta V, Nikolic B, Kalluri R, Carmeliet P, Mundel P, Reiser J: Modification of kidney barrier function by the urokinase receptor. Nat Med 2008, 14(1):55-63.

15. Stephens RW, Nielsen HJ, Christensen IJ, Thorlacius-Ussing O, Sorensen S, Dano K, Brunner N: Plasma urokinase receptor levels in patients with colorectal cancer: relationship to prognosis. J Natl Cancer Inst 1999, 91(10):869-874.

16. Konno H, Baba M, Shoji T, Ohta M, Suzuki S, Nakamura S: Cyclooxygenase-2 expression correlates with uPAR levels and is responsible for poor prognosis of colorectal cancer. Clin Exp Metastasis 2002, 19(6):527-534.

17. Wei C, El Hindi S, Li J, Fornoni A, Goes N, Sageshima J, Maiguel D, Karumanchi SA, Yap HK, Saleem M, Zhang Q, Nikolic B, Chaudhuri A Daftarian P, Salido E, Torres A, Salifu M, Sarwal MM, Schaefer F, Morath C, Schwenger V, Zeier M, Gupta V, Roth D, Rastaldi MP, Burke G, Ruiz P, Reiser $\mathrm{J}$ : Circulating urokinase receptor as a cause of focal segmental glomerulosclerosis. Nat Med 2011, 17(8):952-960.

18. Riisbro R, Christensen IJ, Piironen T, Greenall M, Larsen B, Stephens RW, Han C, Hoyer-Hansen G, Smith K, Brunner N, Harris AL: Prognostic significance of soluble urokinase plasminogen activator receptor in serum and cytosol of tumor tissue from patients with primary breast cancer. Clin Cancer Res 2002, 8(5):1132-1141.

19. Carmeliet $P$, Moons L, Lijnen $R$, Baes $M$, Lemaitre $V$, Tipping $P$, Drew $A$, Eeckhout Y, Shapiro S, Lupu F, Collen D: Urokinase-generated plasmin activates matrix metalloproteinases during aneurysm formation. Nat Genet 1997, 17(4):439-444.

20. Wei Y, Lukashev M, Simon DI, Bodary SC, Rosenberg S, Doyle MV, Chapman HA: Regulation of integrin function by the urokinase receptor. Science 1996, 273(5281):1551-1555.

21. Madsen CD, Ferraris GM, Andolfo A, Cunningham O, Sidenius N: uPARinduced cell adhesion and migration: vitronectin provides the key. J Cell Biol 2007, 177(5):927-939.

22. Wei Y, Waltz DA, Rao N, Drummond RJ, Rosenberg S, Chapman HA: Identification of the urokinase receptor as an adhesion receptor for vitronectin. J Biol Chem 1994, 269(51):32380-32388.

23. De Bock CE, Lin Z, Mekkawy AH, Byrne JA, Wang Y: Interaction between urokinase receptor and heat shock protein MRJ enhances cell adhesion. Int J Oncol 2010, 36(5):1155-1163.

24. Dai YS, Xu J, Molkentin JD: The DnaJ-related factor Mrj interacts with nuclear factor of activated T cells c3 and mediates transcriptional repression through class II histone deacetylase recruitment. Mol Cell Biol 2005, 25(22):9936-9948.

25. Kelley WL: The J-domain family and the recruitment of chaperone power. Trends Biochem Sci 1998, 23(6):222-227.

26. Calderwood SK, Khaleque MA, Sawyer DB, Ciocca DR: Heat shock proteins in cancer: chaperones of tumorigenesis. Trends Biochem Sci 2006, 31(3):164-172

27. Wang $Y$, Liang $X$, Wu S, Murrell GA, Doe WF: Inhibition of colon cancer metastasis by a $3^{\prime}$ - end antisense urokinase receptor mRNA in a nude mouse model. Int J Cancer 2001, 92(2):257-262.

28. Zhuang H, Jiang W, Zhang X, Qiu F, Gan Z, Cheng W, Zhang J, Guan S, Tang B, Huang Q, Wu X, Huang X, Jiang W, Hu Q, Lu M, Hua ZC: Suppression of HSP70 expression sensitizes NSCLC cell lines to TRAlL-induced apoptosis by upregulating DR4 and DR5 and downregulating c-FLIP-L expressions. J Mol Med (Berl) 2013, 91(2):219-235.

29. Liang X, Yang X, Tang Y, Zhou H, Liu X, Xiao L, Gao J, Mao Z: RNAimediated downregulation of urokinase plasminogen activator receptor inhibits proliferation, adhesion, migration and invasion in oral cancer cells. Oral Oncol 2008, 44(12):1172-1180

30. Gondi CS, Lakka SS, Dinh DH, Olivero WC, Gujrati M, Rao JS: RNAi-mediated inhibition of cathepsin B and UPAR leads to decreased cell invasion, angiogenesis and tumor growth in gliomas. Oncogene 2004 23(52):8486-8496.

31. Zhou $H$, Tang $Y$, Liang $X$, Yang $X$, Yang J, Zhu G, Zheng M, Zhang C: RNAi targeting urokinase-type plasminogen activator receptor inhibits metastasis and progression of oral squamous cell carcinoma in vivo. Int J Cancer 2009, 125(2):453-462.

32. Gondi CS, Kandhukuri N, Dinh DH, Gujrati M, Rao JS: Down-regulation of UPAR and UPA activates caspase-mediated apoptosis and inhibits the PI3K/AKT pathway. Int J Oncol 2007, 31(1):19-27.
33. Vial E, Sahai E, Marshall CJ: ERK-MAPK signaling coordinately regulates activity of Rac1 and RhoA for tumor cell motility. Cancer Cell 2003, 4(1):67-79.

34. Nguyen DH, Catling AD, Webb DJ, Sankovic M, Walker LA, Somlyo AV, Weber MJ, Gonias SL: Myosin light chain kinase functions downstream of Ras/ERK to promote migration of urokinase-type plasminogen activator-stimulated cells in an integrin-selective manner. J Cell Bio/ 1999, 146(1):149-164.

35. Jo M, Thomas KS, O'Donnell DM, Gonias SL: Epidermal growth factor receptor-dependent and -independent cell-signaling pathways originating from the urokinase receptor. J Biol Chem 2003, 278(3):1642-1646.

36. Nguyen DH, Webb DJ, Catling AD, Song Q, Dhakephalkar A, Weber MJ Ravichandran KS, Gonias SL: Urokinase-type plasminogen activator stimulates the Ras/Extracellular signal-regulated kinase (ERK) signaling pathway and MCF-7 cell migration by a mechanism that requires focal adhesion kinase, Src, and Shc. Rapid dissociation of GRB2/Sps-Shc complex is associated with the transient phosphorylation of ERK in urokinase-treated cells. J Biol Chem 2000, 275(25):19382-19388.

37. Doshi BM, Hightower LE, Lee J: The role of Hsp27 and actin in the regulation of movement in human cancer cells responding to heat shock. Cell Stress Chaperones 2009, 14(5):445-457.

38. Olson D, Pollanen J, Hoyer-Hansen G, Ronne E, Sakaguchi K, Wun TC, Appella E, Dano K, Blasi F: Internalization of the urokinase-plasminogen activator inhibitor type-1 complex is mediated by the urokinase receptor. J Biol Chem 1992, 267(13):9129-9133.

39. Cubellis MV, Wun TC, Blasi F: Receptor-mediated internalization and degradation of urokinase is caused by its specific inhibitor PAI-1. Embo J 1990, 9(4):1079-1085.

40. Fazioli F, Blasi F: Urokinase-type plasminogen activator and its receptor: new targets for anti-metastatic therapy? Trends Pharmacol Sci 1994, 15(1):25-29.

41. Arispe N, Doh M, De Maio A: Lipid interaction differentiates the constitutive and stress-induced heat shock proteins Hsc70 and Hsp70. Cell Stress Chaperones 2002, 7(4):330-338.

42. Kanse SM, Kost C, Wilhelm OG, Andreasen PA, Preissner KT: The urokinase receptor is a major vitronectin-binding protein on endothelial cells. Exp Cell Res 1996, 224(2):344-353.

43. Waltz DA, Natkin LR, Fujita RM, Wei Y, Chapman HA: Plasmin and plasminogen activator inhibitor type 1 promote cellular motility by regulating the interaction between the urokinase receptor and vitronectin. J Clin Invest 1997, 100(1):58-67.

44. Stahl A, Mueller BM: Melanoma cell migration on vitronectin: regulation by components of the plasminogen activation system. Int J Cancer 1997, 71(1):116-122

45. Sitrin RG, Todd RF 3rd, Albrecht E, Gyetko MR: The urokinase receptor (CD87) facilitates CD11b/CD18-mediated adhesion of human monocytes. J Clin Invest 1996, 97(8):1942-1951.

46. Boroughs LK, Antonyak MA, Johnson JL, Cerione RA: A unique role for heat shock protein 70 and its binding partner tissue transglutaminase in cancer cell migration. J Biol Chem 2011, 286(43):37094-37107.

47. Sims JD, McCready J, Jay DG: Extracellular heat shock protein (Hsp)70 and Hsp90alpha assist in matrix metalloproteinase-2 activation and breast cancer cell migration and invasion. PLoS One 2011, 6(4):e18848.

48. Sidenius $N$, Blasi F: The urokinase plasminogen activator system in cancer: recent advances and implication for prognosis and therapy. Cancer Metastasis Rev 2003, 22(2-3):205-222.

doi:10.1186/1471-2407-14-639

Cite this article as: Lin et al:: Heat shock proteins HSP70 and MRJ cooperatively regulate cell adhesion and migration through urokinase receptor. BMC Cancer 2014 14:639. 